\title{
Client-Server versus Peer-to-Peer
}

\author{
Başak Altan* \\ Özyeğin University
}

\author{
M. Oğuz Sunay** \\ Özyeğin University
}

\begin{abstract}
This study identifies optimal transmission mechanisms for a video streaming service in a peer-to-peer network structure under different payment mechanisms: pay as you watch and pay upfront. We calculate a uniform, feasible service price using the utilities of the users and associated server profits for every possible peer-to-peer distribution tree. We prove that when the server has linear or concave costs, the client-server structure is more profitable than any peer-to-peer structure. This statement holds even when the users have maximal tolerance to indefinitely long pre-roll delays. When the server however, has convex costs, we show that the optimal network structure depends on the system parameters and there is no single distribution mechanism that provides the optimal server profit for every operating point of the network.
\end{abstract}

Keywords: communication networks, peer-to-peer networks, economics of the Internet, video streaming, pricing. JEL Classification: L11, L86

\section{İstemci-Sunucu'ya karşılık Eşler Arası}

\section{Özet}

Bu çalışma, eşler arası ağ yapısı altında farklı ödeme mekanizmaları (izlerken öde ve önceden öde) ile bir video akışı hizmeti için en iyi dağıtım mekanizmalarını belirlemektedir. Kullanıcıların faydaları ve sunucunun bunlara denk gelen karlarını kullanarak her bir olası eşler arası dağıtım ağacı için tekdüze olurlu bir fiyat hesaplanmaktadır. Sunucunun doğrusal veya içbükey maliyetlere sahip olması durumunda, istemci-sunucu yapısının, herhangi bir eşler arası yapıya göre daha karlı olduğunu ispatlanmıştır. Bu ifade kullanıcıların yayın öncesi gecikmelere karşı azami toleransa sahip oldukları durumda dahi geçerliliğini korumaktadır. Sunucunun dişbükey maliyetlere sahip olduğu durumda ise en iyi ağ yapısının sistem parametrelerine bağlı olduğu ve ağın her bir işletim noktası için en çok sunucu karını sağlayan tek bir dağıtım mekanizmasının olmadı̆̆ı gösterilmiştir.

Anahtar Kelimeler: iletişim ağları, eşler arası ağlar, internet ekonomisi, video akışı, fiyatlandırma.

JEL Sinıflamasi: L11, L86

* Başak Altan is an Assistant Professor in the Department of Economics at Özyeğin University, Nişantepe Mahallesi, Orman Sokak, Çekmeköy, 34794, Istanbul, Turkey. E-mail: basak.altan@ozyegin.edu.tr

** M. Oğuz Sunay is an Associate Professor in the Department of Electrical Engineering at Özyeğin University, Nişantepe Mahallesi, Orman Sokak, Çekmeköy, 34794, Istanbul, Turkey. E-mail: oguz.sunay@ozyegin.edu.tr 
treaming media is the most significant contributor to the Internet traffic. A multimedia is constantly delivered by a provider to the end-users. According to the technical report by Cisco Systems Inc., IP video traffic is expected to make up $80 \%$ of all IP traffic by 2019 , up from $67 \%$ in 2014 . To understand the magnitude of this volume, the required time to watch the amount of video that will cross global IP networks each month in 2019 is 5 million years. Internet TV is a common form of streamed media. It is expected that Internet video TV will grow at a very rapid pace, fourfold, and will constitute 17\% of consumer Internet video traffic in 2019 (Cisco Systems Inc., 2015). There are two possible transmission mechanisms for live or ondemand video streaming services: client-server $(\mathrm{CS})$ and peer-to-peer $(\mathrm{P} 2 \mathrm{P})$. In the CS mechanism, the server provides the video directly to all the end-users, whereas in any P2P mechanism, the server provides the video to a subset of end-users and then these users seed the content to other end-users. The commercial P2P streaming TV services that enable users to watch live or non-live are free of charge (Fowler G. and McBride S (2005)). The commercial streaming service BBC iPlayer that is built upon a P2P mechanism with a pay as you watch pricing scheme has failed. BBC iPlayer has been using a client-server system since 2008. This observation is in line with the results of our study where we identify conditions for which a P2P distribution is the optimal mechanism.

In this study, we considered a live system to distribute video streams in real time. These systems are significant as they allow a scalable distribution of video streams to a large audience. There are many commercial P2PTV applications (PPLive, PPStream, LiveStation.com, etc.). Many of these services are quite active in China (Fowler G. and McBride S. (2005)). Peer-to-peer assisted streaming services turn a regular user into a re-broadcaster of service with no additional cost to the server. The system that we consider is similar to a P2PTV where if the system dictates, a peer uploads the content while downloading it. Hence, we are not interested in the incentives for sharing even if it is costly to share. The server, however, has to consider the cost of sharing while pricing the service. In this study, we identify the profit maximizing mechanism to stream multimedia content with a linear price under different cost structures of the service provider and different payment mechanisms. We identify whether it is optimal to distribute the content in one stage (CS) or in multiple stages (P2P). We also establish the optimal $\mathrm{P} 2 \mathrm{P}$ propagation tree structure: how many stages and how many users at each stage. We consider two different payment mechanisms: pay as you watch and pay upfront.

There is a vast amount of literature on the technical perspective of CS and P2P videostreaming mechanisms. Yong et al. (2008) and Zhang and Hassanein (2012) review different approaches from the technical perspective of network structures. The literature on the economic perspective is, however, scarce. The seminal work by Walrand (2008) introduces the concept of a closely inter-linked "technology layer" and "economic layer" for communication systems to correctly model the inter-play between a profitable communication service and the underlying technology providing it. The studies that consider the interplay between the technology and economic layers for P2P systems in the literature are usually focused on incentive mechanisms to maximize peer participation. Wu et al. (2012) and Chu et al. (2009) consider a video on-demand service where incentives are 
provided via auction-based re-pricing strategies for seeding users. A similar, auctionbased approach is presented in Tan and Jarvis (2008) for a streaming broadcast service. Park and van der Schaar (2010) investigate incentive schemes to overcome the free-rider problem in content production and sharing and to show that a cooperative outcome can be achieved among non-cooperative peers by introducing an incentive scheme based on pricing, reciprocation, or intervention. A game theoretic framework is proposed in Lin et al. (2009) to model the peer behavior in a P2P live streaming system and it is used to design incentive schemes to encourage peer participation. Asioli et al. (2012) develop a game theoretic model for scalable video streaming with an incentive mechanism to enable optimal resource allocation in a P2P network. Finally, Altan and Sunay (2016) identify the optimal network structure under both static and dynamic participation of peers where the server collects the service fee in advance. Our study is also related to pricing mechanisms to allocate the transmission capacity to end-users (MacKie-Mason and Varian, 1995a; MacKie-Mason and Varian, 1995b; Shenker et al., 1996; Gupta et al., 1997; Gupta et al., 2000; Mason, 2000). However, our attention is particularly on the optimal distribution structure and the price under two different pricing mechanisms: pay as you watch and pay upfront.

We establish that, regardless of the payment structure, the CS mechanism is more profitable than any $\mathrm{P} 2 \mathrm{P}$ mechanism unless the cost of the server is convex in the number of users. This statement holds even when the users have maximal tolerance to indefinitely long pre-roll delays (the time it takes to buffer and to begin playing the video after a user makes a request to download it). When the cost of the server is convex, we observe that the optimal mechanism depends on the system parameters, the curvature of the cost function and the payment mechanism.

The remainder of this paper is organized as follows. Section 2 discusses the model. A mathematical analysis is discussed in Section 3. Section 4 presents corresponding results for different user participation structures. Finally, conclusions are drawn in Section 5.

\section{The Model}

We consider a system with a server and $N$ users with identical hardware capabilities and Internet connectivity. We assume that the server and all users are risk neutral. The server streams a multimedia broadcast, a video, to these users. The video is distributed through a sequentially ordered series of equal sized chunks. None of the users leave the system unless they receive all the video chunks. As illustrated in Figure 1, out of $N$ users in the system, $N_{1}$ of them are assumed to be served directly by the server and the remaining users receive the broadcast service over an $s$-stage spanning tree $\left\langle N_{1}, N_{2}, \ldots, N_{s}\right\rangle$ with $\sum_{i=1}^{S} N_{i}=N$ where $N_{i}$ is the number of users receiving the video in stage $i$. If the video distribution mechanism is CS then $N_{j}=0$, for all $j \geq 2$. When the server directly broadcasts the content to $N_{1}$ users, its total cost is represented by $C\left(N_{1}\right)$. We assume that $C(1)=c$ and that there is no restriction on the curvature of the server costs. 
Figure 1

\section{P2P Streaming Multimedia Broadcast Model}

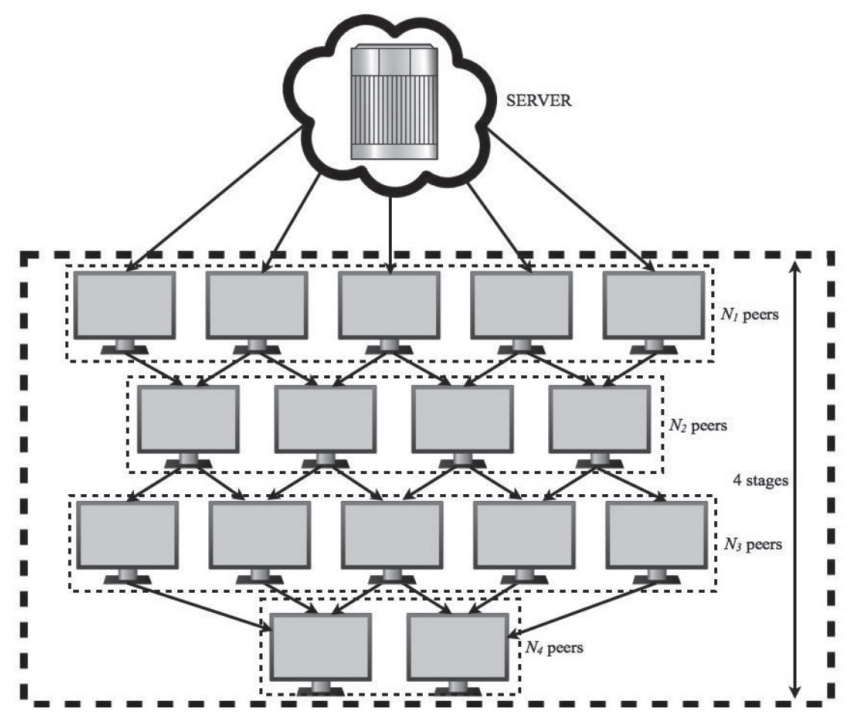

The reservation utility of a user for the privilege of watching the broadcast is $\theta>0$ with

$$
\theta \equiv \int_{0}^{l} v \exp (-r n) d n
$$

where $v$ is the flow benefit that a user derives from the video service, $r$ is the discount rate, and $l$ is the length of the video.

We assume that the accrued cost of a user when she is downloading a video chunk from either the server or another user is $c{ }^{[1]}$ Additionally, the accrued cost of a user when she is seeding the content to another user is $c$ as well. We also assume that the cost of parallel seeds at the same stage is linear in the number of parallel seeds. A user may upload (seed) the content for the use of other users. If a user is supposed to seed the content to a subset of other users, we assume that download and uploads occur at the subsequent stages. If a user receives the video at stage $t$ and seeds the content to $n$-many users at stage $t+1$, her total costs become $\beta^{t}(1+\beta) c$ where $\beta \equiv e^{-r z}$ is the discount factor and $z$ is the time period between two successive stages.

While pricing the service, the server considers the end-user with the lowest net utility. We refer to this user as the marginal user. When the server charges a price that results in a negative utility for the marginal user, it would prefer not purchasing the service. Hence, as all users have identical valuations and identical upload/download costs before

[1] Our results survive even if the accrued cost of a user when she is downloading a video chunk from another user is higher than the accrued cost when she is downloading it from the server. 
discounting, the price of the service cannot be set any higher than the indifference price for the marginal buyer. We consider two different payment models in this paper: pay as you watch (PAYW) and pay upfront (PUF) payment schemes. In the PAYW scheme, users pay for the viewing of a stream exactly at the start of their playback. In other words, a user that has a non-zero pre-roll delay pays for the service later than a user that has none. In the PUF scheme on the other hand, all users, regardless of their pre-roll delays, pay for the service upfront, when the video is first introduced.

Next, we develop the system model for the two uniform payment models considered: pay as you watch (PAYW) and pay upfront (PUF).

\section{Pay as You Watch Payment Model}

Suppose that the uniform price of the video is set to be . In the PAYW model, the server derives a net profit of

$$
\Pi=N_{1} p_{1}-C\left(N_{1}\right)+\sum_{t=2}^{\infty} N_{t} \beta^{t-1} p_{1} .
$$

In the profit equation above, the first term is the total revenue from the set of $N_{1}$ users directly receiving the video stream from the server in the first period. These users do not experience any multi-hop pre-roll delay. Therefore, the server collects the fees from these users right away and thus no discounting is necessary. The second term is the cost of the server accumulated from providing the video stream to this first set of users. The last term represents discounted revenues from the remaining set of users receiving the video from another user. Since a user's playback start time is modeled to be linearly proportional to its stage location in the $\mathrm{P} 2 \mathrm{P}$ tree, and since a user pays the server upon the start of playback in the PAYW payment scheme, the revenues accrued from these users are discounted at a level that is dependent on their stage locations in the spanning tree. Note that the price, $p_{1}$ has to be set as the indifference price of the marginal user since uniform payment is employed.

In the PAYW payment model, a user that starts viewing the video stream in period $t_{1}$ and starts seeding it to one other user in the next period derives a net utility of

$$
U=\beta^{t_{1}}\left(\theta-p_{1}-c\right)-\beta^{t_{1}+1} c .
$$

The first term, $\beta^{t_{1}} \theta$ is the discounted service valuation obtained by receiving the stream $t_{1}$ periods later than the present time. The associated costs for this delivery are the payment, $\beta^{t_{1}} p_{1}$ as well as the cost of downloading, $\beta^{t_{1}} c$, both discounted since they occur, similar to the valuation term, $t_{1}$ slots later than the present time. The cost of seeding the stream to one other user is the last term in the utility equation above, which is equal to $\beta^{t_{1}+1} c$, discounted since it occurs $t_{1}+1$ slots later than the present time.

Let us define $U(i)$ as the utility of a user starting the video playback in period $i$. In this case, when the video if distributed via an s-stage spanning tree mechanism

$$
U(i)=\left\{\begin{array}{cc}
\beta^{i-1}\left(\theta-c-p_{1}-\beta \alpha_{i} c\right), & i=1, \ldots, s-1, \\
\beta^{i-1}\left(\theta-c-p_{1}\right), & i=s
\end{array}\right.
$$


where $\alpha_{i}$ is the probability that the user aids in the propagation of the video chunk under investigation. The maximum price that can be set has to ensure that the utilities of all of the users remain nonnegative. In that case,

$$
p_{1}=\theta-c\left(1+\beta \max \left\{\alpha_{i}\right\}\right) \text {. }
$$

\section{Pay Upfront Payment Model}

Suppose now that the uniform price is set to be $p_{2}$. The server's net profit in the PUF payment model is then equal to

$$
\Pi=\sum_{t=1}^{\infty} N_{t} p_{2}-C\left(N_{1}\right) .
$$

Since the server collects the service payments upfront, regardless of when the stream is delivered to users, there is no discounting on any of the received payments, nor is there one for the cost associated with delivering the stream directly to $N_{1}$ users since they both take place in the present time.

Similarly, under the PUF payment model, a user that starts viewing the video stream in period $t_{1}$ and starts seeding it to one other user in the next period derives a net utility of

$$
U=\beta^{t_{1}}(\theta-c)-p_{2}-\beta^{t_{1}+1} c .
$$

Unlike the utility of the same user when the PAYW payment model is used, the price paid for the service is not discounted here as it is paid in advance at the start of subscription. The service value and the cost of downloading and seeding, on the other hand, are discounted using the appropriate discounting factors.

Let us define $\mathrm{U}(\mathrm{i})$ as the utility of a user starting the video playback in period i. In this case, when the video is distributed via an s-stage spanning tree mechanism

$$
U(i)=\left\{\begin{array}{cc}
\beta^{i-1}(\theta-c)-p_{2}-\beta^{i} \alpha_{i} c, & i=1, \ldots, s-1, \\
\beta^{i-1}(\theta-c)-p_{2}, & i=s
\end{array}\right.
$$

where $\alpha_{i}$ is the probability that the user aids in the propagation of the video chunk under investigation. Then the maximum price that can be set is equal to

$$
\begin{gathered}
p_{2}=\min \left[\theta-c\left(1+\beta \alpha_{1}\right), \beta\left(\theta-c\left(1+\beta \alpha_{2}\right)\right), \ldots,\right. \\
\left.\beta^{s-1}\left(\theta-c\left(1+\beta \alpha_{s-1}\right)\right), \beta^{s}(\theta-c)\right] .
\end{gathered}
$$

For both of the cost models we focus on the nontrivial case where $\theta>2 c$. Otherwise, it is clear that the server has no incentive to transmit the video to a user.

\section{Analysis}

The fundamental aim of this paper is to identify the most profitable video streaming distribution mechanism over the Internet. As such, the goal is to identify how many 
stages the spanning tree should have, how many users should reside in each stage of the tree, and what the individual share ratios of the users should be.

For a given population, $N$, we first characterize the pricing behavior of the server for every possible peer-to-peer network configuration, including, as a special case, the clientserver mechanism. The streaming video price is equal to the marginal user indifference price for each spanning tree configuration. We then formulate the server profit, taking into account the revenues, as well as costs accumulated for serving a subset of the users directly.

\section{Client-Server Network}

In CS, the server streams the video directly to a subset of users, $n_{C S} \leq N$, that maximizes its profit in chunks of equal size. Depending on the costs of the server, which are a function of the serviced user population, a value less than $N$ may yield the highest profit. Since the CS network is a single stage spanning tree, every user belongs to the first stage and thus has the same utility which is equal to

$$
U_{1}=\theta-P_{C S}-c \text {. }
$$

A user's utility term includes the service value, the price paid for it, as well as the cost of downloading. It should be noted that this utility is valid for both PAYW and PUF payment schemes since the CS scheme is a single stage spanning tree where every user receives the stream directly from the server. Since all users have identical utilities, all are in fact marginal users. Then, the price of the service is set as their indifference price, which is equal to

$$
P_{C S}=\theta-c
$$

and the resulting profit of the server from streaming the video to $n_{C S}$ users is

$$
\Pi_{C S}=n_{C S} P_{C S}-C\left(n_{C S}\right) \text {. }
$$

It is clear that the server strictly prefers streaming the video to the entire user community (i.e. $n_{C S}=N$ ) when costs are linear or concave. When costs are convex, however, the number of users for whom the server transmits the video for optimal profit depends on the curvature of the cost function.

\section{Peer-to-Peer Network}

In P2P, the server initially streams the video to a subset of users. Then, a user may stream the video chunks to other users. In this paper, we place no limit on how many parallel seeds a user may provide. However, it should be noted that as the number of seeds increases for a user, its utility decreases due to increasing costs. There are a very large number of possible P2P distribution trees. However, when the PAYW payment scheme is used, the profit wise optimal P2P distribution tree needs to satisfy the following lemma:

Lemma 1. For a video distribution to users, all seeding users in the P2P spanning tree should behave identically, i.e. they should all seed with the same share ratio, $\alpha$, when the PAYW payment scheme is employed. 
Proof. In video transmission, consider two users that receive the same video chunk at any time. Consider that they seed chunks from the stream in the following period with share ratios $\alpha^{\prime}$ and $\alpha^{\prime \prime}$ respectively. Then, the price the server can charge for the video is

$$
P=\theta-\left(1+\beta \max \left\{\alpha^{\prime}, \alpha^{\prime \prime}\right\}\right) c .
$$

When the share ratios of all other users are unchanged, $\alpha^{\prime}+\alpha^{\prime \prime}$ should be maintained at a constant value to maintain an uninterrupted service to the population of $N$. In this case, the above-stated price is maximized when the share ratio for each of these two users is $\frac{\alpha^{\prime}+\alpha^{\prime \prime}}{2}$. Since the net payoffs of two users in different stages are discounted by $\beta$, all users must share with the same ratio, $\alpha$, at the profit maximizing video distribution mechanism under a PAYW payment scheme.

Lemma 1 means that the maximum profitable P2P spanning tree should have the property,

$$
\alpha=\frac{N_{2}}{N_{1}}=\frac{N_{3}}{N_{2}}=\cdots=\frac{N_{S}}{N_{S-1}} .
$$

Then, we represent a candidate P2P spanning tree for optimal profitability as $\left\langle N_{1}, N_{2}, \ldots, N_{s}\right\rangle$ with common per-stage user share ratios of $\left(\alpha_{1}, \alpha_{2}, \ldots, \alpha_{s}\right)$ where $\alpha_{1}=\alpha_{2}=\cdots=\alpha_{s}=\alpha$ when the PAYW payment scheme is considered. In this network, the server directly streams video chunks to $N_{1}$ users, which all seed with a common share ratio of $\alpha_{1}$ to $N_{2}$ users in the second stage of the spanning tree. Similarly, these $N_{2}$ users seed with a share ratio of $\alpha_{2}$ to $N_{3}$ users in the third stage of the spanning tree, and so on. Then, in a network structure with $N$ subscribers,

$$
N=N_{1}\left(1+\sum_{i=2}^{s} \prod_{j=1}^{i-1} \alpha_{j}\right) .
$$

When the PAYW payment scheme is used, $N=N_{1}\left(1+\sum_{i=2}^{S} \prod_{j=1}^{i-1} \alpha_{j}\right)$ simplifies to

$$
N=N_{1}\left(1+\sum_{i=2}^{s} \alpha^{i-1}\right) .
$$

Let us first consider the PAYW payment model. To find the uniform price, $P_{P 2 P}$, of the streaming service we need to find the utilities of all users in the system. Lemma 1 states that the share ratios of all users should be identical for the most profitable P2P tree. Then, the utilities of all users in the same stage of the tree will be the same. Then, the maximum uniform price the server can charge for the streaming video service is equal to,

$$
P_{P 2 P}=\theta-(1+\beta \alpha) c .
$$

Note here that the P2P streaming video service price decreases with an increasing discount factor and an increasing common share ratio under the PAYW payment scheme. 
The corresponding profit of the $\mathrm{P} 2 \mathrm{P}$ spanning tree under investigation is then equal to

$$
\Pi=N_{1} P_{P 2 P}-C\left(N_{1}\right)+N_{1} \sum_{i=1}^{s-1}(\beta \alpha)^{i} P_{P 2 P} .
$$

In the profit equation above, the first term represents the revenue collected from the $N_{1}$ users that are served directly by the server. The second term is the associated costs of streaming the video to these users. The third term is the discounted revenues collected from all the other users in the network who are seeded the video from other users. Note here that the P2P spanning tree with $N_{1}=N, N_{2}=\cdots=N_{s}=0$ is the CS mechanism. It is clear that the PAYW price and profit become equal to the CS price and profit respectively, in this case.

Similarly, when the PUF payment scheme is used, the optimal P2P distribution tree needs to satisfy the following lemma:

Lemma 2. For a video distribution to users, all seeding users that are in the same stage of the P2P spanning tree should behave identically, i.e. users in stage $i$ of the spanning tree should all seed with the same share ratio, $\alpha_{i}$ when the PUF payment scheme is employed.

Proof. Consider two users from stage $i$ of the P2P tree with share ratios of $\alpha^{\prime}$ and $\alpha^{\prime \prime}$, respectively. Their utilities are, $\beta^{i}\left(\theta-\left(1+\beta \alpha^{\prime}\right) c\right)-p$ and $\beta^{i}\left(\theta-\left(1+\beta \alpha^{\prime \prime}\right) c\right)-p$, respectively. If the price is determined by one of the stage $i$ users, it is equal to,

$$
P=\beta^{i}\left(\theta-\left(1+\beta \max \left\{\alpha^{\prime}, \alpha^{\prime \prime}\right\}\right) c\right) .
$$

When the share ratios of all other users in the $i$ 'th stage are unchanged, $\alpha^{\prime}+\alpha^{\prime \prime}$ should be maintained at a constant value to maintain an uninterrupted service to the users in the $i+1$ 'th stage. Then, the above stated price is maximized when the share ratio for each of these two users is $\frac{\alpha^{\prime}+\alpha^{\prime \prime}}{2}$. Since different users in different stages pay the fee of the video service at the beginning of the stage they are in, we can only conclude that users in the same stage of the spanning tree should all seed with the same share ratio. This lemma means that

$$
\alpha_{i}=\frac{N_{i+1}}{N_{i}}, i \in\{1,2, \ldots, s-1\}
$$

is the share ratio for all users that are located in the $i$ 'th stage of the spanning tree. Next, we consider the PUF payment model. We determine the utilities of all of the users to find the uniform price, $P_{P 2 P}$, the server can set for the service. As with the PAYW payment scheme, when the PUF payment scheme is employed, the per-stage utilities are once again the same. Let us define

$$
\gamma_{i}=\theta-\left(1+\beta \alpha_{i}\right) c \text {. }
$$


Then, the maximum uniform price the server can charge for the streaming video service is equal to

$$
P_{P 2 P}=\min \left\{\gamma_{1}, \beta \gamma_{2}, \ldots, \beta^{s-2} \gamma_{s-1}, \beta^{s-1}(\theta-c)\right\} .
$$

The corresponding profit of the $\mathrm{P} 2 \mathrm{P}$ spanning tree is then equal to

$$
\Pi=N P_{P 2 P}-C\left(N_{1}\right) .
$$

In the profit equation above, the first term represents the revenue collected from all $N$ users who pay for the service upfront. Therefore these payments do not experience any discounting. The second term is the associated costs of streaming the video directly to $N_{1}$ users that are in the first stage of the P2P propagation tree.

\section{Results}

Based on the profit terms derived in the previous section, we now identify the optimal network structure as a function of $N, \beta$ and $c$ under different cost structures of the server $C(\cdot)$. We consider linear costs $C^{\prime}(n)>0$ and $C^{\prime \prime}(n)=0$, concave costs $C^{\prime}(n)>0$ and $C^{\prime \prime}(n)<0$, and convex costs $C^{\prime}(n)>0$ and $C^{\prime \prime}(n)>0$ where we assume for all cost structures $C(1)=c$ without any loss of generality. There are a large number of possible spanning trees for the streaming video service even for the modest user populations. In this paper, we provide a mathematical framework that takes all of these distribution schemes into account.

We observe that when the server costs are a linear or concave function of the number of users that are directly served by the server, the most profitable distribution scheme is the CS structure, regardless of the value of the discount factor and the payment scheme employed. This observation is valid even when the users have a maximum tolerance to pre-roll delays. In other words, even when the users have a maximum tolerance to waiting indefinitely for the stream playback to start, the CS scheme is still more profitable than any P2P network. This is because when P2P is employed the server has to take the users' costs of seeding into account while establishing a price for the service. However, the corresponding revenue drop observed in $\mathrm{P} 2 \mathrm{P}$ mechanisms relative to the CS mechanism is never sufficiently compensated by the drop in the server costs for the P2P mechanisms if linear or concave server costs are observed.

On the other hand, when the server costs are a convex function of the number of users that are directly served by the server, we observe that there is no dominant distribution structure. Different distribution schemes become most profitable for different discount factors. We now prove these observations and illustrate them using examples.

Theorem 1. For maximum profit, the streaming video server must transmit the video using the CS mechanism if its cost structure is linear or concave and the PAYW payment scheme is employed. 
Proof. When costs are linear, the profits from CS and P2P become

$$
\Pi_{C S}=N(\theta-2 c) \text {, }
$$

and

$$
\begin{gathered}
\Pi_{P 2 P}=N_{1}\left(1+\beta \alpha+\cdots+(\beta \alpha)^{s-1}\right)(\theta-2 c) \\
-N_{1}(\beta \alpha)^{s} c
\end{gathered}
$$

respectively. Since $N=N_{1}\left(1+\sum_{i=2}^{S} \alpha^{i-1}\right)$ holds for the PAYW payment scheme and $\beta \in[0,1]$, we must have

$$
N_{1}\left(1+\beta \alpha+\cdots+(\beta \alpha)^{s-1}\right) \leq N .
$$

Hence, the server prefers CS to P2P, that is

$$
\Pi_{C S} \geq \Pi_{P 2 P} .
$$

Moreover, if the CS scheme is preferred with linear costs, it is also preferred with concave costs due to economies of scale.

Figure 2

Server Profits of all Possible P2P Tree Structures as a Function of the Discount Factor when $N=10$, PAYW is Employed and Server Costs Follow a Linear Function

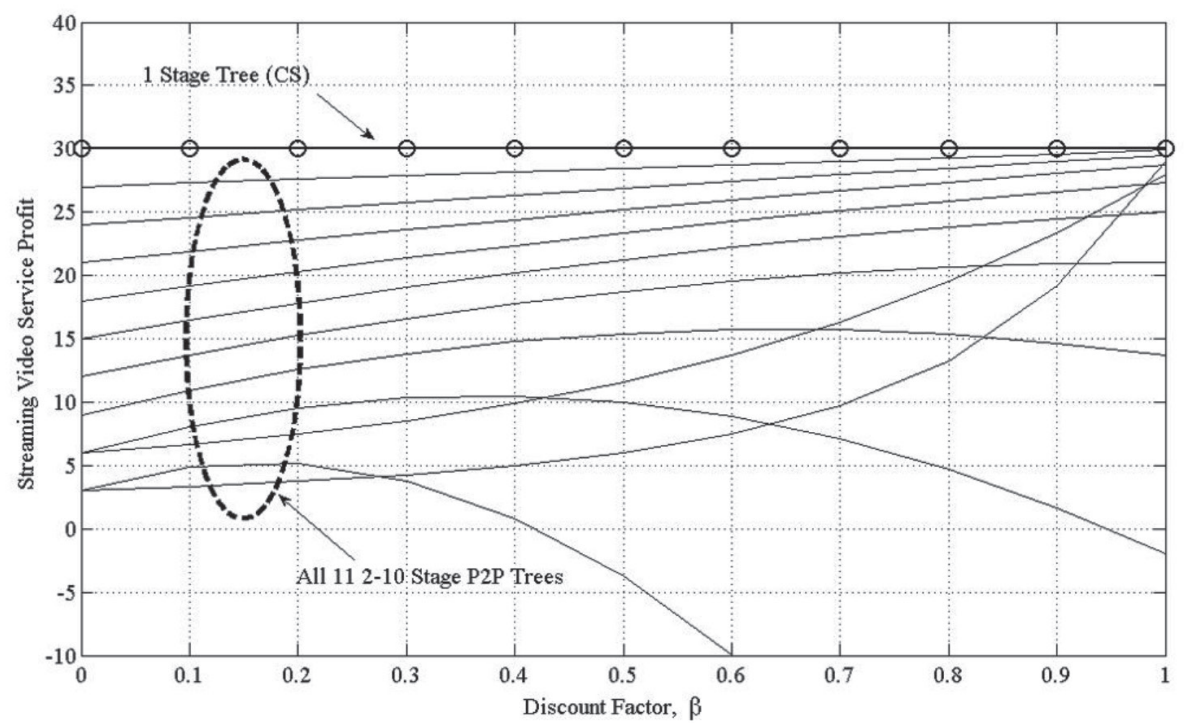

Example 1. To illustrate Theorem 1, let us consider a streaming video service with a population of ten users. It is known that when the PAYW payment scheme is employed the spanning tree in use should obey Lemma 1 for the service to be maximally profitable. There are a total of 12 spanning trees, 
one representing the $C S$ and the rest representing $P 2 P$ schemes that satisfy Lemma 1. Of the 11 P2P trees, 9 of them have two stages, 1 has five stages and 1 has 10 stages. We consider a linear function for $C(\cdot)$ and let the ratio of service value to the cost of downloading/uploading a single stream, $\theta / c=5$. We calculate the server profits for different discount factor, $\beta$, values for the 12 spanning trees. The results are illustrated in Figure 2. For each $\beta$ value, we also calculate the highest profit any $i$-stage spanning tree provides. The results are illustrated in Figure 3. The results confirm the statement we make in Theorem 1. We observe that all P2P mechanisms are less profitable than the CS mechanism. As $\beta$ increases towards 1, it is clear that the most profitable P2P mechanism approaches profit in the CS mechanism. However, even when $\beta=1$, which models the case where users are tolerant to indefinitely long durations of pre-roll delays, the CS mechanism is at least as profitable as the most profitable P2P mechanism. Furthermore, we observe from Figure 3 that for any $\beta$ value, the most profitable 2-stage spanning tree is always at least as profitable as the 5-stage and 10-stage spanning trees that are longer.

Figure 3

Highest Server Profits from all Possible Stages in the P2P Tree as a Function of the Discount Factor when $N=10$, PAYW is Employed and Server Costs Follow a Linear Function

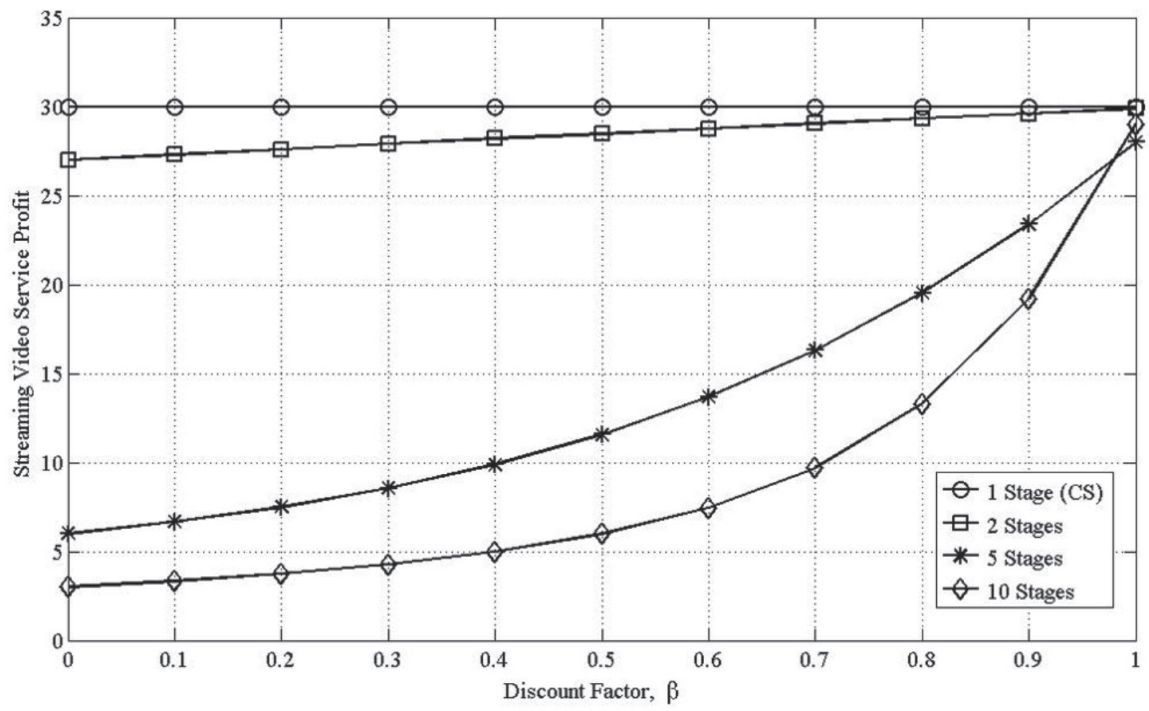

Proposition 1. When the server has convex costs, there is no dominant transmission strategy for the server in the grand set of all possible P2P propagation trees with per-stage user populations of $\left\langle N_{1}, N_{2}, \ldots, N_{s}\right\rangle$ and corresponding universal user share ratio of $r$ when the PAYW payment scheme is employed. 
Proof. We prove by a counter example: Consider a system with 4 users. There are a total of 8 possible different $\mathrm{P} 2 \mathrm{P}$ propagation tree structures: $\langle 4\rangle<3,1><2,2><1,3\rangle$ $<2,1,1><1,2,1><1,1,2>$ and $<1,1,1,1>$. Out of the 8 trees, 3 of them do not obey Lemma 1: $<2,1,1><1,2,1>$ and $<1,1,2>$. Therefore they have no possibility of being the most profitable transmission strategy. We consider a simple convex cost function of $C(\cdot)=(\cdot)^{2}$, and a service value to a single download cost ratio, $\theta / c$, of 10 . The profits drawn from these transmission strategies, including the ones that do not obey Lemma 1 , are plotted as a function of the discount factor, $\beta$ in Figure 4.

Figure 4

Server Profits as a Function of the Discount Factor when $N=4$, PAYW is Employed and Server Costs Follow the $(\cdot)^{2}$ Function

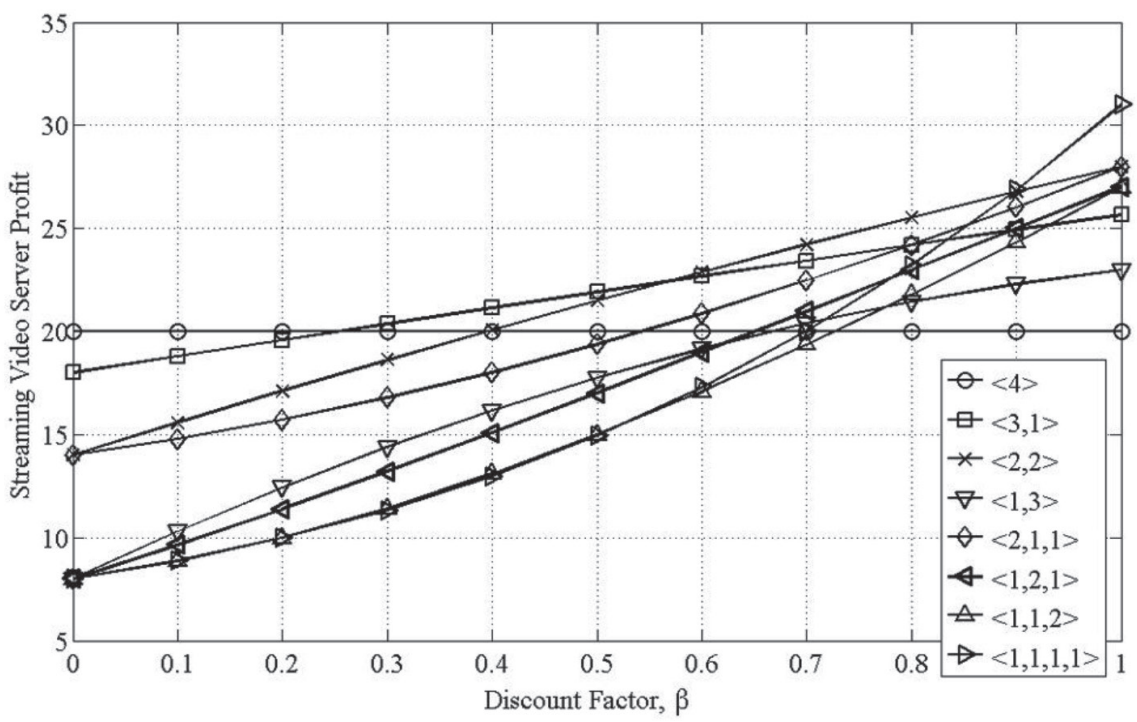

From Figure 4 we observe that for different discount factor values, different transmission strategies provide the highest service profit. When $\beta$ is less than 0.25 , the CS scheme is the most profitable. However, as the users become more tolerant of their pre-roll delays, different $\mathrm{P} 2 \mathrm{P}$ strategies become more profitable. Which one of the $\mathrm{P} 2 \mathrm{P}$ trees is optimum depends on how tolerant users are to their pre-roll delays. Therefore, there is no dominant distribution strategy when the server costs are a convex function of the number of users served directly by the server.

Theorem 2. For maximum profit, the streaming video server must transmit the video using the CS mechanism if its cost structure is linear or concave and the PUF payment scheme is employed. 
Proof. Let us first assume that the discount factor is equal to $\beta=1$. In other words, consider a system where users are maximally tolerant to pre-roll delays. In this case, when the costs are linear, the profits from CS and P2P become

$$
\Pi_{C S}=N(\theta-2 c)
$$

and

$$
\Pi_{P 2 P}=N\left(\theta-\left(1+\mu+\frac{N_{1}}{N}\right) c\right)
$$

respectively, where

$$
\mu=\max _{i=1, \ldots, s-1}\left\{\alpha_{i}\right\}
$$

is the maximum share ratio in the $\mathrm{P} 2 \mathrm{P}$ tree. From $\Pi_{C S}$ and $\Pi_{P 2 P}$ it is clear that the CS mechanism is strictly preferred to any $\mathrm{P} 2 \mathrm{P}$ mechanism if and only if

$$
\mu+\frac{N_{1}}{N} \geq 1
$$

We now prove that this is indeed the case using mathematical induction.

Consider first a P2P tree composed of only the bottom two stages of the generalized layout illustrated in Figure 1. In this case, $N=N_{s-1}+N_{s}, N_{1}=N_{s-1}$ and $\mu=N_{s} / N_{s-1}$. Then, we have $\mu+\frac{N_{1}}{N}=\frac{N_{S}}{N_{S-1}}+\frac{N_{S-1}}{N}$ since, $N=N_{s-1}+N_{s}, \mu+\frac{N_{1}}{N}$, is equal to $\frac{N_{S}}{N_{S-1}}+\frac{N_{S-1}}{N_{S-1}+N_{S}}$. Then, $\frac{N_{S}}{N_{S-1}}+\frac{N_{S-1}}{N_{S-1}+N_{S}}=\frac{N_{S-1}^{2}+N_{S-1} N_{S}+N_{S}^{2}}{N_{S-1}^{2}+N_{S-1} N_{S}}$. Therefore, it is clear that $\mu+\frac{N_{1}}{N} \geq 1$ is always true.

Now consider the bottom three stages of the generalized layout of Figure 1. In this case, $N=N_{s-2}+N_{s-1}+N_{s}, N_{1}=N_{s-2}$ and $\mu=\max \left\{N_{s} / N_{s-1}, N_{s-1} / N_{s-2}\right\}$. Now, if

$$
\frac{N_{s-1}}{N_{s-2}} \geq \frac{N_{s}}{N_{s-1}}
$$

then $\mu+\frac{N_{1}}{N} \geq 1$ becomes

$$
\frac{N_{s-1}}{N_{s-2}}+\frac{N_{s-2}}{N_{s-2}+N_{s-1}+N_{s}} \geq 1 \text {, }
$$

which always holds. If on the other hand, 


$$
\frac{N_{s-1}}{N_{s-2}} \leq \frac{N_{s}}{N_{s-1}}
$$

then $\mu+\frac{N_{1}}{N} \geq 1$ becomes

$$
\frac{N_{s}}{N_{s-1}}+\frac{N_{s-2}}{N_{s-2}+N_{s-1}+N_{s}} \geq 1,
$$

which once again always holds.

Next, consider the bottom $s-1$ stages of the generalized layout of Figure 1. In this case, $N=N_{2}+\cdots+N_{S}$, and $N_{1}=N_{2}$. Furthermore, let

$$
\xi=\max _{i=2, \ldots, S}\left\{\alpha_{i}\right\}
$$

Then $\mu+\frac{N_{1}}{N} \geq 1$ becomes

$$
\xi+\frac{N_{2}}{N_{2}+\cdots+N_{s}} \geq 1 .
$$

We assume that this inequality holds.

Last, we consider all $s$ stages of the generalized layout of Figure 3. Here,

$$
\mu=\max \left\{\xi, \frac{N_{2}}{N_{1}}\right\}
$$

We consider both possibilities for the value of $\mu$. First, let us assume that

$$
\xi \geq \frac{N_{2}}{N_{1}}
$$

is true. Then $\mu+\frac{N_{1}}{N} \geq 1$ becomes

$$
\xi+\frac{N_{1}}{N_{1}+\cdots+N_{s}} \geq 1 .
$$

It is straightforward to observe that this always holds due to our assumption

$$
\text { and } \xi \geq \frac{N_{2}}{N_{1}} \text {. }
$$

Now, let us assume that

$$
\xi \leq \frac{N_{2}}{N_{1}}
$$

holds. Using $\xi=\max _{i=2, \ldots, s}\left\{\alpha_{i}\right\}$, this is equivalent to stating that 


$$
\frac{N_{2}}{N_{1}} \geq \frac{N_{3}}{N_{2}}, \frac{N_{2}}{N_{1}} \geq \frac{N_{4}}{N_{3}}, \cdots, \frac{N_{2}}{N_{1}} \geq \frac{N_{s}}{N_{s-1}}
$$

is true. Then $\mu+\frac{N_{1}}{N} \geq 1$ becomes

$$
\frac{N_{2}}{N_{1}}+\frac{N_{1}}{N_{1}+\cdots+N_{s}} \geq 1,
$$

which always holds due to $\frac{N_{2}}{N_{1}} \geq \frac{N_{3}}{N_{2}}, \frac{N_{2}}{N_{1}} \geq \frac{N_{4}}{N_{3}}, \cdots, \frac{N_{2}}{N_{1}} \geq \frac{N_{S}}{N_{S-1}}$. Therefore, we prove that the CS mechanism is strictly more profitable than any P2P mechanism for the PUF payment scheme when $\beta=1$ and the cost structure is linear. In this case, the CS scheme is also the preferred scheme with concave costs due to economies of scale.

If $\mathrm{CS}$ is strictly preferred to $\mathrm{P} 2 \mathrm{P}$ scheme when $\beta=1$ as proven above, we can deduce that it is indeed the preferred scheme for any value of $\beta \in[0,1]$ if the profits of the server increase as $\beta$ increases. We now prove that $\frac{\partial \Pi_{P 2 P}}{\partial \beta}>0$. The price of the s-stage $\mathrm{P} 2 \mathrm{P}$ system is $\min \left\{\theta-\left(1+\beta \alpha_{1}\right) c, \beta\left(\theta-\left(1+\beta \alpha_{2}\right) c\right), \ldots, \beta^{s-2}\left(\theta-\left(1+\beta \alpha_{s-1}\right) c\right)\right.$, $\left.\beta^{s-1}(\theta-c)\right\}$ where $\alpha_{i}=\frac{N_{i+1}}{N_{i}}$ for all $i$. At the optimal $\left(N_{1}^{*}, \ldots N_{s}^{*}\right)$, we must have $\theta-\left(1+\beta \alpha_{1}\right) c=\beta\left(\theta-\left(1+\beta \alpha_{2}\right) c\right)=\cdots=\beta^{s-2}\left(\theta-\left(1+\beta \alpha_{s-1}\right) c\right)=\beta^{s-1}(\theta-$ $c)$. Otherwise, we can readjust $\alpha_{i}, i \in\{1, \ldots s-1\}$ and either decrease costs or increase the price, or both. Then if the costs are linear, the profit of the optimal s-stage P2P system becomes $\Pi_{P 2 P}=N \beta^{s-1}(\theta-c)-c N_{1}^{*}$. Let us represent the optimal $N_{1}$ for an s-stage P2P system as $N_{1}^{*}(s)$. We will prove that $\frac{\partial N_{1}^{*}(s)}{\partial \beta}<N(s-1) \beta^{s-2} \frac{\theta-c}{c}$ for all $s$. Let us define $N_{1}^{*}(s)$ :

$N_{1}^{*}(s)=\frac{c \beta a_{s-1}}{(\theta-c)\left(1-\beta^{s-1}\right)+c \beta a_{s-1}}$

where $a_{i}=\frac{c \beta a_{i-1}}{(\theta-c)\left(1-\beta^{i-1}\right)+c \beta a_{i-1}}$ for all $i \in\{2,3, \ldots\}$ and $a_{1}=1$. This implies that we have, $\frac{\partial N_{1}^{*}(s)}{\partial \beta}=N(\theta-c) \beta^{s-2} c^{s-1} f(\beta, \theta, \mathrm{c}, \mathrm{s})$ where $\frac{\partial f(\beta, \theta, \mathrm{c}, \mathrm{s})}{\partial \beta}<0$ and $\frac{\partial f(\beta, \theta, \mathrm{c}, \mathrm{s})}{\partial \theta}<0$.

Then, we can show that $f(\beta, \theta, \mathrm{c}, \mathrm{s})<\frac{s-1}{c^{s}}$ holds. Hence, $\frac{\partial N_{1}^{*}(s)}{\partial \beta}<N(s-1) \beta^{s-2} \frac{\theta-c}{c}$ also holds.

The profit generated by the CS system is no less than the profit generated by all $\mathrm{P} 2 \mathrm{P}$ systems when $\beta=1$. Additionally, the profit generated by P2P systems decreases as $\beta$ decreases, whereas the profit generated by the CS system does not change with 
respect to $\beta$. Thus, we establish that the CS system generates more profit than any $\mathrm{P} 2 \mathrm{P}$ system for any $\beta$.

Example 2. To illustrate Theorem 2, let us reconsider the streaming video service of Example 1 with 10 users. The maximally profitable spanning trees need to obey Lemma 2 when the PUF payment scheme is employed. There are a total of 512 such spanning trees. Once again we consider a linear function for $C(\cdot)$ and $\theta / c=5$. Using Using $\Pi_{C S}$ and $\Pi_{P 2 P}$, we calculate the server profits as a function of $\beta$ for all of the spanning trees. The results are illustrated in Figure 5.

Figure 5

Server Profits of all Possible P2P Tree Structures as a Function of the Discount Factor when $N=10$ PUF is Employed and Server Costs Follow a Linear Function

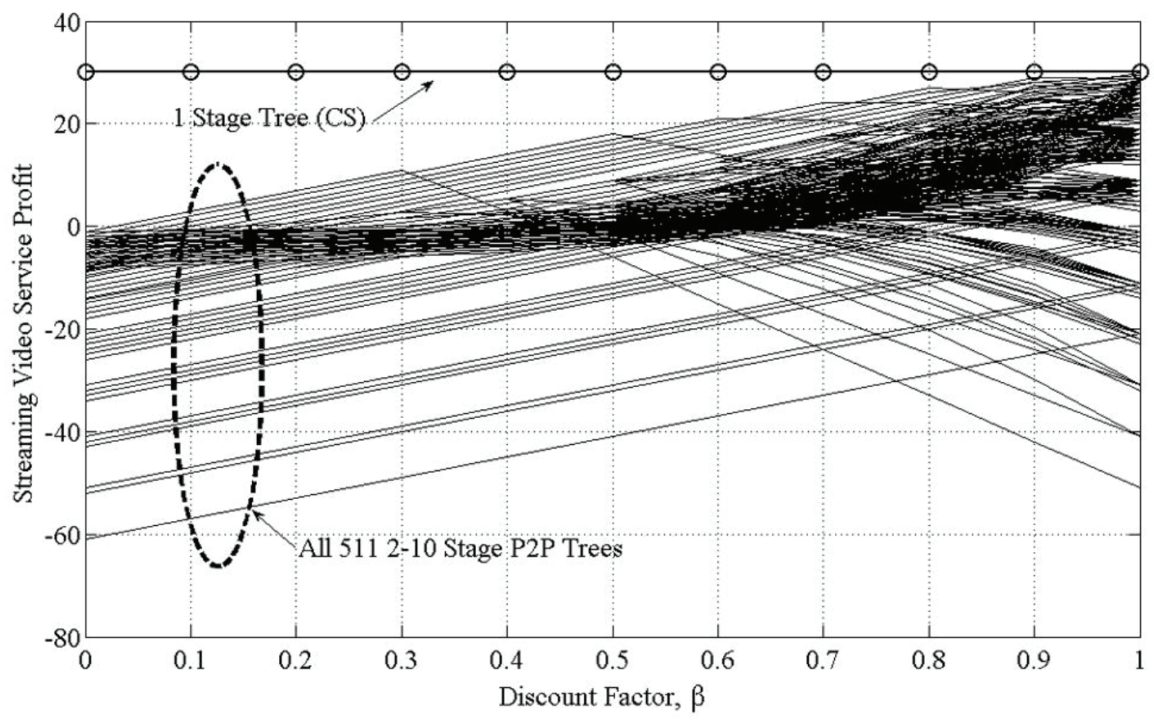

For each $\beta$ value, we also calculate the highest profit any stage $i$ spanning tree provides. The results are illustrated in Figure 6. The results confirm the statement of Theorem 2. As with the PAYW payment scheme, we observe that when the PUF payment scheme is employed all P2P mechanisms are less profitable than the CS mechanism for any value of $\beta$. Increasing $\beta$ values increase the profitability of $P 2 P$ transmission. However, once again, even when $\beta=1$, which models the case where users are tolerant to indefinitely long durations of pre-roll delays, the CS mechanism is at least as profitable as the most profitable $P 2 P$ mechanism, as in the PAYW pricing mechanism. Furthermore, we observe from Figure 6 that for any $\beta$ value, the most profitable 2-stage spanning tree is always at least as profitable as all other trees that are longer. 
Figure 6

Highest Server Profits from all Possible Stages in the P2P Tree as a Function of the Discount Factor when $\boldsymbol{N}=10$ PUF is Employed and Server Costs Follow a Linear Function

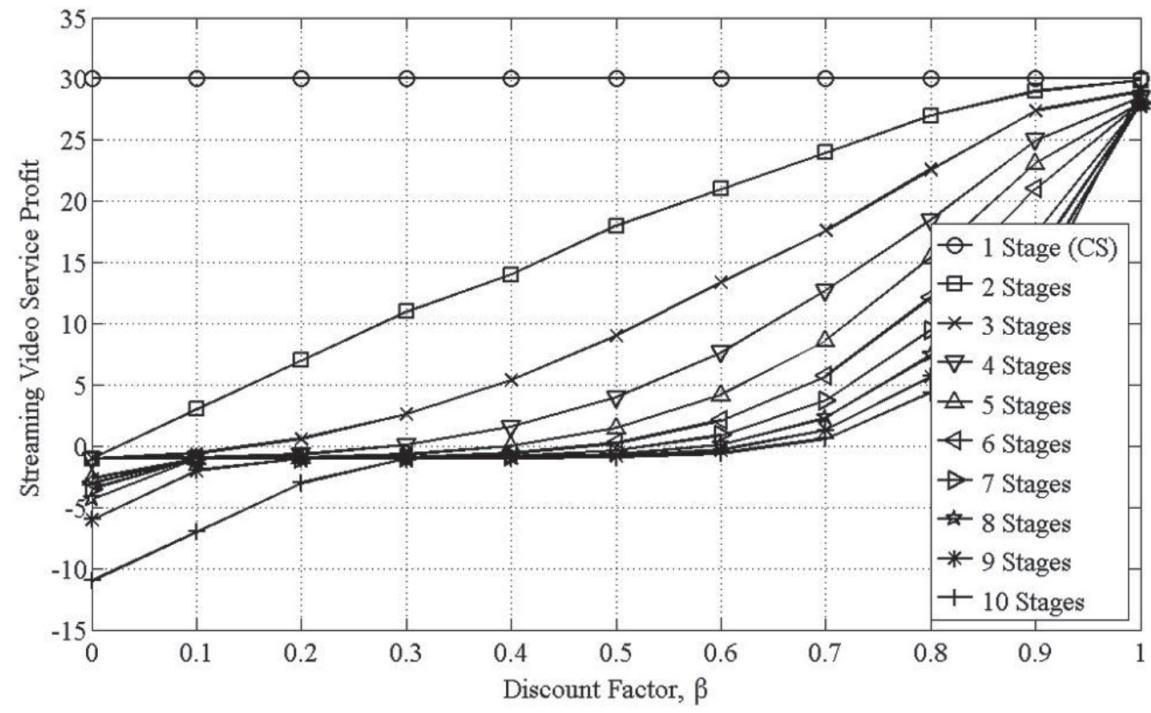

Proposition 2. When the server has convex costs, there is no dominant transmission strategy for the server in the grand set of all possible P2P propagation trees with per-stage user populations of $\left\langle N_{1}, N_{2}, \ldots, N_{S}\right\rangle$ and corresponding per-stage user share ratios of $\left\langle r_{1}, r_{2}, \ldots, r_{s}\right\rangle$ when a PUF payment scheme is employed.

Proof. As with Proposition 1, we prove by a counter example. Consider the same system with 4 users discussed in the proof of Proposition 1 with a total of 8 possible different $\mathrm{P} 2 \mathrm{P}$ propagation tree structures. Once again we consider a simple convex cost function of $C(\cdot)=(\cdot)^{2}$, and a service value to single download cost ratio, $\theta / c$, of 10 . The profits drawn from these transmission strategies are plotted as a function of the discount factor, $\beta$ in Figure 7.

From Figure 7 we observe that, as with the case of the PAYW payment scheme, when the PUF scheme is employed, for different discount factor values, different transmission strategies provide the highest service profit. We observe that the CS scheme is the most profitable for values of $\beta$ less than 0.58 in this scenario. However, as the discount factor increases, different P2P strategies become more profitable than the CS architecture. Therefore, the most profitable distribution mechanism depends on the curvature of the cost function, the $\beta$ and $\theta / c$ values when the server costs are a convex function of the number of users served directly by the server. 
Figure 7

\section{Server Profits as a Function of the Discount Factor when $N=4$, PUF is Employed and} Server Costs Follow the $(\cdot)^{2}$ Function

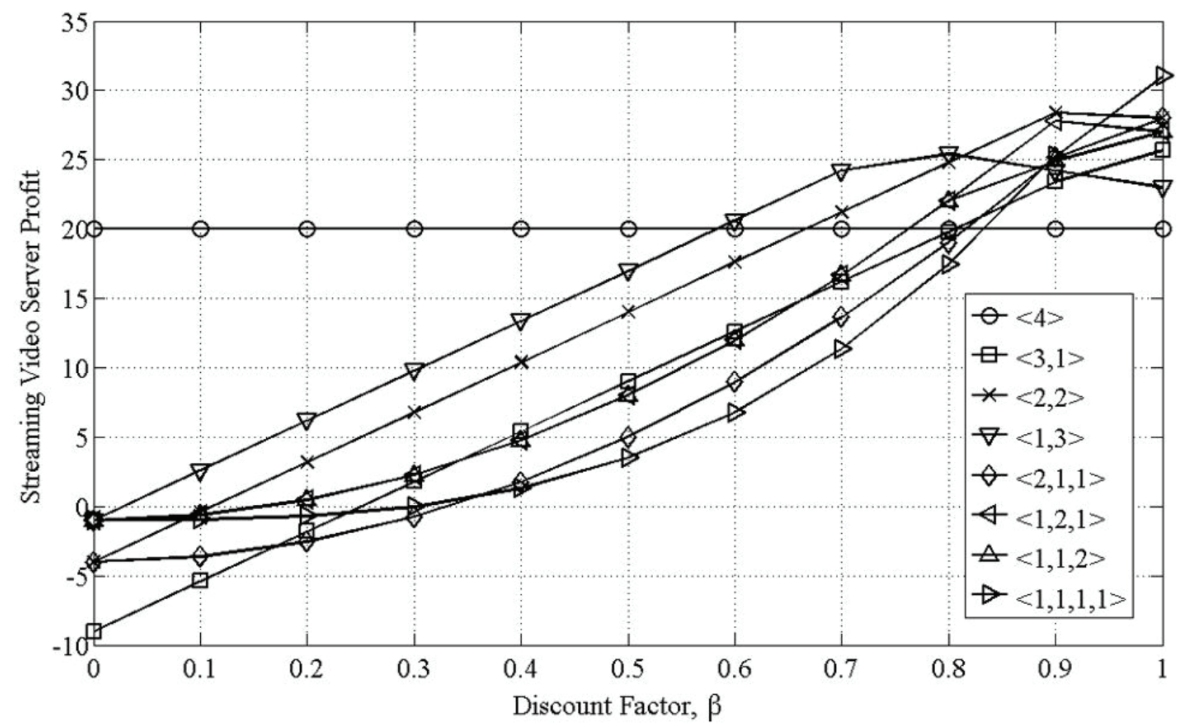

\section{Conclusion}

In this study, we identify optimal transmission mechanisms for a streaming video application in a peer-to-peer network structure as a function of the number of active users, a common service value, a common discount factor, downloading and seeding costs of a user for a single video stream, and the cost structure of the server. We consider two different payment mechanisms: the pay as you watch mechanism where a user pays for the service at the time of the stream's playback starting time, and the pay upfront mechanism where all users pay for the service in advance. We calculate the common service price using the users' utilities. We then derive the server profits for every possible P2P distribution tree. When the streaming video server employs a common pricing policy, the price that can be set for the service cannot be any larger than the indifference price of the marginal user. Otherwise, certain users would end up with negative utilities and therefore would not subscribe to the service. When P2P is used as a distribution mechanism, the price that can be set has to take the users' video download and seeding costs into account. In such a framework, we prove that when the server has linear or concave costs, the optimal transmission mechanism is the server-client structure. This statement is true even when the users have maximal tolerance to indefinitely long preroll delays. The main reason behind this conclusion is that the price reduction due to the CS mechanism one observes in P2P mechanisms due to the cost of seeding is always 
larger than the reduction in the server costs when some of the users are served by other users instead of the server. We prove that this conclusion holds for both the pay as you watch and pay upfront payment schemes. When the server, however, has convex costs, we prove that the optimal network structure depends on the system parameters and that there is no one distribution mechanism that provides the optimal server profit for every operating point of the network

\section{References}

Altan, B. and Sunay, M.O. (2016). "Optimal Peer-to-Peer Network for Streaming Multimedia Broadcast," Working Paper.

Asioli, S., Ramzan, R. and Izquierdo, E. (2012). “A Game Theoretic Approach to Minimum-Delay Scalable Video Transmission Over P2P,” Signal Processing: Image Communication, 27: 513-521.

Chu, X., Zhao, K., Li Z,. and Mahanti, A. (2009). "Auction-Based On-Demand P2P Min-Cost Media Streaming with Network Coding," IEEE Transactions on Parallel and Distributed Systems, 20: 1816-1829.

Fowler, G. and McBride, S. (2005). "Newest Export From China: Pirated Content," The Wall Street Journal, September 2, B1.

Gupta, A., Stahl, D., and Whinston, A. (1997). "A Stochastic Equilibrium Model of Internet Pricing," Journal of Economic Dynamics and Control, 21: 697-722.

------, (2000). "Extracting Consumers' Private Information for Implementing Incentive Compatible Internet Traffic Pricing," Journal of Management Information Systems, 17: 9-29.

Lin, W.S., Zhao, H.V., and Liu, K.J.R. (2009). "Incentive Cooperation Strategies for Peer-to-Peer Live Multimedia Streaming Social Networks,” IEEE Transactions on Multimedia, 11: 396-412.

MacKie-Mason, J.K. and Varian, H. (1995a). "Pricing Congestible Network Resources," IEEE Journal of Selected Areas in Communication, 13: 1141-1149.

-----, (1995b). "Pricing the Internet," in B. Kahin and J. Keller (eds.), Public Access to the Internet: 269-314. Prentice Hall, Englewood Cliffs.

Mason, R. (2000). “Simple Competitive Internet Pricing,” European Economic Review, 44: 1045-1056.

Cisco Systems Inc. (2015). “The Zettabyte Era-Trends and Analysis,” Tech. rep., Cisco Systems Inc.

Park, J. and van der Schaar, M. (2010). "A Game Theoretic Analysis of Incentives in Content Production and Sharing Over Peer-to-Peer Networks," IEEE Journal on Selected Topics in Signal Processing, 4: 704-717.

Shenker, S., Clark, D., Estrin, D., and Herzog, S. (1996). "Pricing in Computer Networks: Reshaping the Research Agenda," Telecommunications Policy, 20: 183-201.

Tan, G. and Jarvis, S.A. (2008). "A Payment-Based Incentive and Service Differentiation Scheme for Peer-to-Peer Streaming Broadcast," IEEE Transactions on Parallel and Distributed Systems, 19: 940-953.

Walrand, J. (2008). "Economic Models of Communication Networks," in Z. Liu and C.H. Xia (eds.), Performance Modeling and Engineering: 57-90. Springer. 
Wu, C., Li, Z., Qiu, X., and Lau, F.C.M. (2012). “Auction-Based P2P VoD Streaming: Incentives and Optimal Scheduling," ACM Transactions on Multimedia Computing, Communications and Applications, 22(15): 14:1-14:22.

Yong, L., Yang, G., and Chao L. (2008). "A Survey on Peer-to-Peer Video Streaming Systems," Peer-to-Peer Networking and Applications, 1: 18-28.

Zhang, X. and Hassanein, H. (2012). “A Survey on Peer-to-Peer Live Video Streaming Schemes - An Algorithmic Perspective," Computer Networks, 56: 3548-3579. 
